\title{
Intra-, Inter-, and Cross-cultural Classification of Vocal Affect
}

\author{
Daniel Neiberg ${ }^{1}$, Petri Laukka ${ }^{2}$, \& Hillary Anger Elfenbein ${ }^{3}$ \\ ${ }^{1}$ Department of Speech, Music and Hearing (TMH), KTH, Stockholm, Sweden \\ ${ }^{2}$ Department of Psychology, Stockholm University, Stockholm, Sweden \\ ${ }^{3}$ Olin Business School, Washington University in St. Louis, St. Louis, MO, USA \\ neiberg@speech.kth.se, petri.laukka@psychology.su.se, helfenbein@wustl.edu
}

\begin{abstract}
We present intra-, inter- and cross-cultural classifications of vocal expressions. Stimuli were selected from the VENEC corpus and consisted of portrayals of 11 emotions, each expressed with 3 levels of intensity. Classification (nu-SVM) was based on acoustic measures related to pitch, intensity, formants, voice source and duration. Results showed that mean recall across emotions was around 2.4-3 times higher than chance level for both intra- and inter-cultural conditions. For cross-cultural conditions, the relative performance dropped $26 \%, 32 \%$, and $34 \%$ for high, medium, and low emotion intensity, respectively. This suggests that intracultural models were more sensitive to mismatched conditions for low emotion intensity. Preliminary results further indicated that recall rate varied as a function of emotion, with lust and sadness showing the smallest performance drops in the crosscultural condition.
\end{abstract}

Index Terms: emotion, affect, cross-cultural

\section{Introduction}

This study addresses benchmarking of automatic affective recognition systems in three conditions: 1 ) when training and evaluation is done within the same culture (intra-cultural), 2) when training and evaluation is done for multiple cultures (inter-cultural), and 3) when training is done on one culture and evaluation is done on another (cross-cultural). The results from such an evaluation may not only determine the strengths and weakness of the classifier and acoustic features, but can also be used to investigate the degree of universality of vocal emotion expressions.

The present study has few direct parallels in the literature, but a couple of studies have investigated similar topics. In [1] and [2], classification experiments using novel acoustic features were conducted for five native languages of India. With sequential signal processing improvements, the authors finally presented recall rates very close to $100 \%$ for both intraand cross-cultural classification, outperforming human listeners. While these studies clearly showed the benefit of the proposed features, few other conclusions could be drawn from the results. In another study [3], inter- and intra-cultural experiments were found to yield similar results. However, no details were offered, so no other conclusions than this fact can be drawn.

A slightly different classification problem is both crosscorpora and cross-cultural experiments. This is more difficult, because various affective corpora not only have different affective categories, but the emotions are acted or elicited under different circumstances, and also recorded under different conditions. One approach to this problem is to translate the various affective categories of each corpus into positive, negative and neutral. This approach was adopted by [4], where 4 natural/spontaneous emotional corpora were used. The best intra-corpus result, in terms of unweighted average recall, was 1.39 times chance while the best cross-corpus result was 1.27 times chance. Few results were significant above chance which makes the results difficult to interpret. In a larger similar study [5], the different affective classes in different corpora were mapped into similar ones, instead of the three-class valence categories. For example, for the Danish Emotional Speech corpus containing acted basic emotions, the intra-cultural results were around 3 times above chance (60\% unweighted average recall for 5 basic emotions) and the crosscultural results were around 1.6 times chance for different numbers of sets (around 33\% for 5 basic emotions).

Another strand of research on cross-cultural emotion recognition consists of perceptual studies. In a comprehensive meta-analysis of intra- and cross-cultural perceptual experiments, results for facial and vocal emotions are summarized and compared [6]. The in-group (i.e., intracultural) advantage for vocal expressions ranged between 7.2$17.5 \%$, in ascending order for anger, sadness, fear, happiness, while results for other categories were too sparsely reported. Also [7], in another meta-analysis using a different selection of studies, reported an in-group advantage of around $7 \%$ for vocal expressions. Again, the smallest in-group advantage was found for anger and sadness, and the largest for happiness. However, several individual studies have also reported that negative emotions are easier to recognize cross-culturally than positive emotions [8][9]. The performance drop for crosscultural conditions can be explained by the degree of linguistic similarity [10], or shaping by salient cultural display rules and social environment. The displayed emotional expression has been theorized to be characterized by the 'push effects' caused by physiological effects of arousal and the 'pull effects' of the social norms which both shape the expression and the recognition of specific emotions [11].

In this study, we aim to conduct inter-, intra- and crosscultural automatic recognition experiments for a selection of 11 affective categories. This paper is arranged as follows: In Section 2 the VENEC corpus is presented; Section 3 describes the acoustic measurements extracted using Praat; Section 4 describes the classification experiments; Section 5 presents the results; the discussion is found in Section 6; and conclusions in Section 7.

\section{The VENEC corpus}

We utilized a selection of emotional utterances taken from the VENEC corpus [12]. One hundred professional actors, twenty from USA, Australia, India, Kenya and Singapore each vocally expressed various emotions (anger, contempt, fear, happiness, interest, lust, pride, relief, sadness, shame, and neutral). All emotions except neutral were expressed in three emotional intensities by enacting various emotion-eliciting situations. The actors were provided with scenarios describing typical situations in which each emotion may be elicited, based on current research on emotion appraisals. The verbal material consisted of one of two short English phrases with 
emotionally neutral content (i.e., "Let me tell you something" or "That's exactly what happened") per expression. Our selection included a total of 3,100 emotional portrayals (5 countries, 20 speakers and 11 emotion categories, 3 emotional intensities, one sample per category).

\section{Acoustic measurements}

The set of acoustic features for automatic classification is a slightly extended set than the set used for analysis in [13]. Out of a larger set, we balanced criteria based on principal components analysis (PCA), intercorrelation and interpretability of solutions. While a closer analysis of the features is not presented here, we intend to explain the results based on on-going statistical analyses. Thirty acoustic measures related to pitch, intensity, formants, voice source and duration were automatically extracted using Praat scripts [14], see Table 1 for a brief description of each acoustic measure. In a preprocessing step, each utterance was segmented into pseudo-syllables, defined as sequences of unvoiced, voiced, and unvoiced segments with intensity and duration above certain thresholds which were tuned for current data. Most acoustic measures were calculated for these pseudo-syllables, and their averages over the whole utterance were then used for machine learning. Some fundamental frequency and intensity based measurements, such as minimum, maximum, range, mean, standard deviation, quantiles and slope were measured over the whole utterance. All pitch based measures use logarithmic scale and all intensity based measures use $\mathrm{dB}$ scale.

\section{Experiments}

Classification experiments were carried out using nu-SVM with radial base kernel from the LibSVM package [15] using default shrinking heuristics. The already reduced size of the feature vector gives very little room for improvement by using feature selection techniques. Three conditions for all three emotional intensities were evaluated: intra-cultural when training and evaluation is done within the same culture; crosscultural when training is done on one culture and evaluation is done on another; and inter-cultural when training and evaluation is done for multiple cultures. Depending on condition, one of three evaluation schemes was chosen, based on the trade-off between execution time and ensuring speakerindependent results. For the intra-cultural condition a leaveone-speaker-out scheme was used whereas for the crosscultural condition a leave-one-culture-out scheme was used (where the latter scheme ensures speaker-independence since the speakers from each culture were different). For the intercultural condition a leave-one-speaker-out-from-eachculture was used, which means that five speakers, each one from different cultures, were used for test and the others were used for training.

For every condition, the regularization parameters (nu and gamma) were optimized using 13 fold cross-validation on each training set. As performance measure we used average recall, defined as the average across the diagonal of the confusion matrix, which is sometimes referred to as unweighted average recall. In addition, the per class recall rates for inter-cultural and cross-cultural condition were more closely examined for the high emotional intensity. We chose to start with the high emotional intensity for this analysis since the push effects are supposed to be maximized while the pull effects are supposed to be minimized. We preferred to measure changes in performance in relative changes since the absolute values are dependent on the classifier, features, number of classes, elicitation procedures etc.

Table 1. Acoustic measures related to pitch, intensity, formants, voice source and temporal aspects of speech.

\begin{tabular}{|l|l|}
\hline Pitch cues \\
\hline F0M/SD & Mean, standard deviation of F0 \\
\hline F0Q1/5 & Quantiles 1/5 of F0 \\
\hline F0Slope & Slope of F0 \\
\hline $\begin{array}{l}\text { F0FracRise/ } \\
\text { Fall }\end{array}$ & Percentage of frames with F0 rise/fall \\
\hline F0SSubtSD & Standard deviation of F0 with the slope subtracted \\
\hline Jitter & $\begin{array}{l}\text { The average absolute difference between } \\
\text { consecutive differences for consecutive periods, } \\
\text { divided by the average period }\end{array}$ \\
\hline Intensity cues \\
\hline IntM/SD & Mean, standard deviation of Intensity \\
\hline IntQ1/5 & Quantiles 1/5 of intensity \\
\hline IntFracRise/ & Percentage of frames with intensity rise/fall \\
\hline Fall & \begin{tabular}{l} 
The average absolute difference between \\
consecutive differences for amplitudes of \\
\hline Shimmer \\
consecutive periods
\end{tabular} \\
\hline Formant cues \\
\hline F1-3M & Mean of formants 1-3 \\
\hline F1-3SD & Standard deviation of formants 1-3 \\
\hline F1-3B & Median bandwidth of formants 1-3 \\
\hline Voice source cues \\
\hline H1MH2 & F0 amplitude-2nd F0 harmonic amplitude \\
\hline H1MA3 & F0 amplitude-formant 3 amplitude \\
\hline Temporal cues \\
\hline SilDurM & Mean of within phrase silence duration \\
\hline SyllDurM & Mean of syllable duration \\
\hline
\end{tabular}

\section{Results}

The intra- and inter-cultural classification results are presented in Table 2. For matched conditions, the following can be observed: The average recall rates are around 2.3-3.0 times above chance (the chance level is $9.1 \%$ ). These numbers are comparable to previous results for this corpus which are 2.6 for 6 classes (basic emotions) and 2.8 for 9 classes (basic and social emotions) [16], as well as results reported for other corpora [5]. By comparing the results for emotional intensities per culture, we observe that 4 of 5 cultures (Aus, Ken, Sin, US) show higher classification performance for medium intensity compared to low intensity. Also, 3 of 5 cultures (Aus, Ind, US) show better classification for high intensity compared to medium intensity, whereas Kenya showed an unexpected drop in recall rate for the high intensity condition. Averaging the recall rates per culture shows a clear trend of increasing average recall when going from low to medium and high intensity, for both intra- and inter cultural training.

Further, our results show little or no advantage for using intra-cultural models compared to intercultural models, without accounting for the larger training size for the intercultural models. However, if Kenya is excluded, there seems to be a small advantage for intra-cultural models. This is consistent with other results [3], and results by [5] where the same system was evaluated on both intra-cultural corpora and the intercultural eNTERFACE corpus. 
Table 2. Results from classification experiments in 3 conditions: intra-cultural (when training and evaluation is done within the same culture), inter-cultural (when training and evaluation is done for multiple cultures), and when training is done on one culture and evaluation is done on another (cross-cultural).

\begin{tabular}{|l|ccc|}
\hline \multicolumn{4}{|c|}{ Intra- and inter-cultural } \\
\hline & high & medium & low \\
\hline Australia & 24.10 & 21.70 & 17.60 \\
\hline India & 34.60 & 27.90 & 28.10 \\
\hline Kenya & 16.30 & 21.30 & 18.40 \\
\hline Singapore & 21.60 & 21.90 & 18.90 \\
\hline US & 28.40 & 26.70 & 24.70 \\
\hline Intra-cult. & 25.00 & 23.90 & 21.54 \\
\hline Intra-cult.* & 27.18 & 24.55 & 22.33 \\
\hline Inter-cultural & 27.10 & 23.40 & 21.40 \\
\hline \multicolumn{4}{|c|}{ Cross-cultural } \\
\hline Australia & 18.50 & 18.80 & 15.50 \\
\hline India & 18.30 & 18.10 & 12.30 \\
\hline Kenya & 18.70 & 13.60 & 15.00 \\
\hline Singapore & 17.70 & 14.00 & 14.60 \\
\hline US & 19.70 & 16.60 \\
\hline Average & 18.58 & 16.22 \\
\hline Average* & 18.55 & 16.08 \\
\hline * excluding Kenya & \multicolumn{3}{c}{14.28} \\
\hline
\end{tabular}

* = excluding Kenya

In the cross-cultural condition, we find quite uniform recall rates for each culture for high emotional intensity, while there is a larger spread for medium and low intensities (see Table 2). The relative drop in average recall rates for the cross-cultural condition is $25.68 \%, 32.13 \%$ and $33.70 \%$ for high, medium and low intensity, respectively. Thus, intracultural models are less sensitive to mismatched cultural conditions for higher emotional intensities.

We also present preliminary findings on recall rates as a function of affective classes for the high emotion intensity condition only (see Table 3). The affective classes which received recall rates larger than the average recall rate are, in descending order: neutral, anger, fear, relief, and happiness for the intra-cultural conditions; and lust, relief, fear, anger, and happiness for the cross-cultural conditions. Thus relief, fear, anger, and happiness appear as the emotions which were easiest to classify under both intra- and cross-cultural conditions. Another picture appears when the relative drop in recall rates for the cross-cultural condition is considered (the average relative drop in recall is $25.68 \%$ ). The classes which show a less than average cross-cultural performance drop (excluding the classes which drop below chance level) are in descending order: lust, sadness, relief and happiness. In particular, lust shows an increase in recall rate and neutral is the worst in this respect.

\section{Discussion}

When comparing the overall system performance for intra-, inter-, and cross-cultural conditions it is clear that changing the condition from intra- to cross-cultural clearly causes a drop in performance. However, this drop is performance is almost completely avoided, regardless of emotional intensity, if intercultural training is performed instead. This finding has implications for the deployment of automatic emotion recognition systems in the field. If it is possible to train intercultural models, then one should always do so, especially if some users are expected to be second language learners. However, different results may be expected if different languages are used in the different cultures, instead of English from different cultural backgrounds.

Table 3. Intra- and cross-cultural recall rates as a function of emotion class for high emotion intensity.

\begin{tabular}{|l|r|r|r|}
\hline & \multicolumn{1}{l|}{ Intra } & \multicolumn{1}{l|}{ Cross } & \multicolumn{1}{l|}{$\begin{array}{l}\text { Relative } \\
\text { difference }\end{array}$} \\
\hline Lust & 24.60 & 30.02 & 22.03 \\
\hline Sadness & 18.90 & 18.10 & -4.23 \\
\hline Relief & 31.80 & 27.70 & -12.89 \\
\hline Pride & 10.10 & 7.88 & -21.98 \\
\hline Happiness & 28.90 & 22.18 & -23.25 \\
\hline Fear & 35.40 & 24.60 & -30.51 \\
\hline Anger & 38.60 & 22.88 & -40.73 \\
\hline Contempt & 24.40 & 13.88 & -43.11 \\
\hline Shame & 13.90 & 7.56 & -45.61 \\
\hline Interest & 21.80 & 11.50 & -47.25 \\
\hline Neutral & 49.40 & 18.16 & -63.24 \\
\hline
\end{tabular}

The performance drop observed when moving from the intra- to the cross-cultural condition may be interpreted in terms of push and pull effects [11]. Whereas the push effects give rise to the emotional expression based on physiological effects, the same expression is also shaped by the social norms, i.e. pull effects, of a group. This can be related to the in-group advantage which is the absolute difference between intra- and cross-cultural recall rates. In [10] the in-group advantage ranged between 5.8-27.8 \% and similar ranges are reported by [6] (only vocal expressions are considered). However, previous documentations of the in-group effect have been based on perceptual experiments, and our results present the first evidence of an in-group advantage using automatic classification based on acoustic features.

It may be assumed that push effects would be particularly salient for expressions with high emotion intensity, whereas pull effects may be more prominent for low emotion intensity expressions. We found that recognition performance was better for higher emotion intensities for the intra-cultural condition. Also, the relative drop in performance seen when moving from intra- to cross-cultural condition was found to be inversely proportional to emotional intensity. These observations tentatively suggest that push effects are proportional to emotion intensity whereas pull effects are inversely proportional to emotion intensity. Further examinations may shed light on the relative strengths or interaction of push and pull effects by, for example, training on one culture and emotion intensity while varying the testing by either changing the culture and emotion intensity in a combinatorial fashion.

We also reported novel classification results for individual affective classes. The classes which were the easiest to recognize in the intra-cultural condition were, in descending order, neutral, anger, fear, relief, and happiness. The highest recall in the cross-cultural condition was instead found for, in descending order, lust, relief, fear, anger and happiness. The latter results are somewhat contradictory to some previous studies based on perceptual experiments. For example [9] reported that anger, sadness and fear were relatively easy to recognize across cultures, though that study only examined six basic emotions (plus neutral) and did not include lust, relief or social emotions. Our results are also somewhat different from [8], who found that negative emotions in general were easier to recognize across cultures. Although the latter included a similar set of affective classes as our study, the study was done on non-lexical vocal outbursts such as laughter and sighs rather than phrases. 
In the present study, the relative drop in recall rate when moving from the intra-cultural to the cross-cultural condition indicated that the acoustic characteristics of lust, sadness, and to some extent relief and happiness were relatively homogenous across cultures, and may thus be least affected by pull effects. These affective classes are somewhat different from the results presented in the surveys of [6] and [7], where anger and sadness showed the smallest, and happiness the largest, in-group advantage. In our study, the social/selfconsciousness emotions pride, contempt, shame and interest showed particularly poor recognition performance under the cross-cultural condition, while at least contempt and interest showed good performance for the intra-cultural condition. This may suggest that social emotions overall are more prone to pull effects than more basic emotions. For speech technology applications, this points toward the importance of using intra-cultural models which include social emotions.

One final caveat that must be kept in mind when comparing the present results with earlier cross-cultural studies is that our study used acoustic features to investigate the acoustic similarity of expressions across cultures, whereas previous documentations of the in-group advantage have been based on perceptual experiments. It may the case that human judges and machines utilize different sets of acoustic cues in order to reach their decisions. For example, human listeners are able to utilize features such as intonation patterns, sentence stress and locations of pauses, which were inaccessible to the automatic classifiers. Also, the statistical inference procedures may bear little similarities with the inference processes involved in human emotion recognition.

\section{Conclusions}

We compared intra-, inter- and cross-cultural classification of affective categories using nu-SVM and a feature set which measures fundamental frequency, intensity, rhythm, voice source and formants. In the intra-cultural condition, training and evaluation was done within the same culture; in the intercultural condition training and evaluation were done for multiple cultures; and in the cross-cultural condition training was done on one culture and evaluation was done on another. Inter-cultural models were found to be almost as efficient as were intra-cultural models (2.4-3 times higher than chance), which means that, if possible, inter-cultural modeling is preferred for speech technology applications. For crosscultural conditions we observed a drop in relative performance, with the largest drop for low emotion intensity (34\%), followed by medium intensity (32\%), and with the smallest drop for high emotion intensity (26\%). Finally, for individual affective classes, relief, fear, anger and happiness had the highest recall rates for both intra- and cross-cultural conditions. Also, lust, sadness, and to some extent relief and happiness, were expressed relatively homogenously across cultures with regard to acoustic features.

Our ongoing work includes: 1) determining the relative strengths and interactions of push and pull effects by examining training on one culture and emotion intensity while varying the testing by either changing the culture or emotion intensity in a combinatorial fashion; 2) to further analyze the classification results for individual affective classes; and 3) to analyze the individual acoustic features as a function of emotion intensity, culture, and affective class.

\section{Acknowledgements}

This work was partly founded by the Swedish Research Council under contracts 2006-1360 and 2009-4599.

\section{References}

[1] Kandali, A. B., Routray, A. and Basu, T. K., "Cross-Lingual Vocal Emotion Recognition in Five Native Languages of Assam Using Eigenvalue Decomposition”, in Pattern Recognition and Machine Intelligence, Lecture Notes in Computer Science, pp. 519-524, 2009.

[2] Kandali, A. B., Routray, A. and Basu, T. K., "Vocal emotion recognition in five native languages of Assam using new wavelet features”, in International Journal of Speech Technology, Vol. 12, no. 1, pp. 1-13, 2009

[3] Origlia, A., Galatà, V. and Ludusan, B., "Automatic classification of emotions via global and local prosodic features on a multilingual emotional database", in Proc. of Speech Prosody 2010, Chicago, 2010.

[4] Eyben, F., Batliner, A., Schuller, B., Seppi D. and Steidl. S. "Cross-Corpus Classification of Realistic Emotions - Some Pilot Experiments”. In: Proc. 7th Intern. Conf. on Language Resources and Evaluation (LREC 2010), Valletta, Malta, May, 2010.

[5] Schuller, B., Vlasenko, B., Eyben, F., Wöllmer, M., Stuhlsatz A., Wendemuth, A. and Rigoll G. "Cross-Corpus Acoustic Emotion Recognition: Variances and Strategies", in IEEE Transactions on Affective Computing (TAC), IEEE, Vol. 1, No. 2, pp. 119-131, July-December, 2010.

[6] Elfenbein, H. A., and Ambady, N. "On the universality and cultural specificity of emotion recognition: A meta-analysis.", in Psychological Bulletin, 128, pp 203-235, 2002.

[7] Juslin, P. N., and Laukka, P. "Communication of emotions in vocal expression and music performance: Different channels, same code?” Psychological Bulletin, 129, pp. 770-814, 2003.

[8] Sauter, D., Eisner, F., Ekman, P., and Scott, S. K. “Cross-cultural recognition of basic emotions through nonverbal emotional vocalizations", in Proceedings of the National Academy of Sciences, 107(6), pp. 2408-2412, 2010.

[9] Pell, M.D., Paulmann, S., Dara, C., Alasseri, A., and Kotz, S.A. "Factors in the recognition of vocally expressed emotions: a comparison of four languages", in Journal of Phonetics, 37, pp 417-435, 2009.

[10] Scherer, K. R., et al. "Emotion inferences from vocal expression correlate across languages and cultures" in Journal of CrossCultural Psychology, 32(1), pp. 76-92, 2001.

[11] Scherer, K. R. "Vocal correlates of emotional arousal and affective disturbance", in Handbook of Social Psychophysiology, H. Wagner and A. Manstead (eds.), pp. 165-197. New York: Wile, 1989.

[12] Laukka, P., Elfenbein, H. A., Chui, W., Thingujam, N. S., Iraki, F. K., Rockstuhl, T., and Althoff, J., "Presenting the VENEC corpus: Development of a cross-cultural corpus of vocal emotion expressions and a novel method of annotating emotion appraisals,” in LREC 2010 Workshop on Corpora for Research on Emotion and Affect, Malta, 2010.

[13] Laukka, P., Neiberg, D., Forsell, M., Karlsson, I., \& Elenius, K. "Expression of Affect in Spontaneous Speech: Acoustic Correlates and Automatic Detection of Irritation and Resignation”, in Computer Speech and Language, 25(1), pp. 84104, 2011.

[14] Boersma, P. and Weenink, D., "Praat: doing phonetics by computer”, 2008, Software available at http://www.praat.org/

[15] Chang, C.-C. and Lin, C.-J., "LIBSVM: a library for support vector machines", 2001, Software available at http://www.csie.ntu.edu.tw/ cjlin/libsvm

[16] Neiberg, D., Laukka, P., and Ananthakrishnan, G. "Classification of Affective Speech using Normalized TimeFrequency Cepstra”, in Prosody 2010, Chicago, USA, 2010. 